\title{
Linguistic-pragmatic factors in interpreting disjunctions
}

\author{
Ira A. Noveck \\ Institut des Sciences Cognitives, Lyon, France \\ Gennaro Chierchia \\ University of Milan-Bicocca, Italy \\ Florelle Chevaux and Raphaëlle Guelminger \\ Institut des Sciences Cognitives, Lyon, France \\ Emmanuel Sylvestre \\ Université de Grenoble, France
}

\begin{abstract}
The connective or can be treated as an inclusive disjunction or else as an exclusive disjunction. Although researchers are aware of this distinction, few have examined the conditions under which each interpretation should be anticipated. Based on linguistic-pragmatic analyses, we assume that interpretations are initially inclusive before either (a) remaining so, or (b) becoming exclusive by way of an implicature (but not both). We point to a class of situations that ought to predispose disjunctions to inclusive interpretations and to situations that encourage exclusive interpretations. A disjunction's ultimate interpretation is based on its potential informativeness, where the interpretation of the disjunctive utterance having the smallest number of true conditions is considered most informative. Our investigation leads to five experiments employing arbitrary materials. Among the problems expected to encourage inclusive interpretations are those that present disjunctions in the antecedents of conditionals and in question forms. The best candidates to produce implicatures are those disjunctions that underdetermine an expected conjunctive conclusion, although other disjunctive utterances that are more informative as exclusive are discussed and tested.
\end{abstract}

Requests for reprints should be sent to Ira Noveck, Institut des Sciences Cognitives, 67 Blvd. Pinel, 69675 Bron, France. Email: noveck@isc.cnrs.fr

This work was supported by a grant from the CNRS (ATIPE, 1999) to the first author. The authors are indebted to Dan Sperber, Guy Politzer, Jean-Baptiste van der Henst, Lewis Bott, Nausicaa Pouscoulous, Phil Johnson-Laird, and Steven Newstead for their valuable insights and for criticisms based on earlier versions of this paper. 
A disjunctive assertion $p$ or $q$ can be understood inclusively (as $p$ or $q$ or both) or exclusively (as $p$ or $q$ but not both). Standard logic introduces a symbol for inclusive disjunctions, $\mathbf{v}$, but few inside and outside the reasoning literature assume that this is the typical way or is treated; as Fillenbaum (1974, p. 913) noted, "or of ordinary usage is ... generally exclusive". Yet, when arbitrary materials are used in reasoning investigations (e.g., letters paired with numbers), participants' natural inclinations concerning or tend towards being inclusive. Investigations with such materials generally report that a majority of participants spontaneously prefer an inclusive interpretation over an exclusive one (Evans \& Newstead, 1980; Paris, 1973) or else equivocality between the two (Braine \& Rumain, 1981). This leaves the reasoning literature open to three questions. (A) If the basic meaning of or indeed tends towards the inclusive, as these studies indicate, how does the exclusive meaning so readily emerge and then appear so dominant in ordinary conversation? (B) If or is typically treated as an exclusive disjunction, why is it so difficult to find that result in standard reasoning tasks that use arbitrary materials? (C) What are (at least some of the factors that prompt a reasoner to interpret or as inclusive or as exclusive?

Before addressing these questions, it is important to appreciate just how effortlessly one can interpret disjunctive utterances. Consider the two realistic disjunctive utterances in (1a) and (1b):
(1) a. An applicant should have a degree in engineering or five years of programming experience.
b. John has an MD or a PhD.

Whereas in (la) an inclusive interpretation is in order, even preferred, in (lb) there appears to be a choice between two options, making the disjunction appear exclusive. This indicates that there must be conditions that determine when an inclusive interpretation should appear salient and when an exclusive interpretation should.

In what follows, we briefly review findings on participants' spontaneous interpretations of $o r{ }^{1}$ We detail a linguistic-pragmatic explanation of the exclusive-or interpretation and show how this account is fruitful for describing existing psychological data. We then take advantage of other insights drawn from linguistic-pragmatic analyses as we examine a set of situations that lead to an inclusive interpretation of $o r$. Finally, we test predictions that follow from our analyses in five experiments.

As Pelletier (1977) and Paris (1973) each pointed out, the only way to determine how people treat or (i.e., as inclusive or exclusive) is to present a

\footnotetext{
${ }^{1}$ We stick to positive contexts in which the disjunction appears unembedded.
} 


\section{TABLE 1}

The truth tables for an exclusive interpretation of the disjunction and for an inclusive disjunction, respectively.

\begin{tabular}{cccc}
\hline$p$ & $q$ & $p$ (exclusive-) or $q$ & $p$ (inclusive-) or $q$ \\
\hline $\mathrm{T}$ & $\mathrm{T}$ & $\mathrm{F}$ & $\mathrm{T}$ \\
$\mathrm{T}$ & $\mathrm{F}$ & $\mathrm{T}$ & $\mathrm{T}$ \\
$\mathrm{F}$ & $\mathrm{T}$ & $\mathrm{T}$ & $\mathrm{T}$ \\
$\mathrm{F}$ & $\mathrm{F}$ & $\mathrm{F}$ & $\mathrm{F}$ \\
\hline
\end{tabular}

situation in which both disjuncts are true. That is, assuming that $p$ and $q$ are both true, an inclusive interpretation of or would lead to an evaluation of $p$ or $q$ as true and an exclusive interpretation would lead to an evaluation of $p$ or $q$ as false; otherwise the truth tables for both sorts of disjunctions are identical (see Table 1). On standard sorts of reasoning tasks that include such situations, adults' spontaneous interpretive tendencies are not entirely consistent. On the one hand, two studies show that adults prefer inclusive interpretations at statistically significant levels. The first comes from Paris (1973) who reported that about $75 \%$ of adults in a developmental study replied true to verbal descriptions like "The boy is riding a bicycle or the dog is lying down" when slides showed both a boy riding a bicycle and a dog lying down (67.5\% reply true when the disjunction is presented as Either ... or). The second comes from Evans and Newstead (1980) who presented rules like "Either there is a P or a 4" and exemplars that varied with respect to the letter or number. In one of their experiments (presented tachistoscopically), participants were forced to respond either true or false to situations in which both disjuncts were true. As Evans and Newstead highlighted, $57 \%$ of the participants replied "true" (and, thus, $43 \%$ replied "false"). Moreover, the authors wrote that True-True exemplars were consistentlyclassified as "true" across three blocks by $57 \%$ of participants and consistently classified as "false" by only $23 \%$. Thus, these authors assumed that the basic interpretation of or tends to be inclusive. On the other hand, Braine and Rumain (1981) report equivocality among adults. They presented (children and) adults with a situation in which a character uttered a statement like "Either there's a cat or there's a frog in the box" when a box contained both; exactly half of the adults said "(the character) was right". When considering a set of utterances that can be classified into response patterns, Braine and Rumain reported that adults tended to prefer the exclusive interpretation (41\%) over an inclusive interpretation (32\%). Thus, at first glance, it would appear that the basic interpretation of or tends to be 
inclusive, but that there is some variability that needs explaining. ${ }^{2}$ We will return to these experimental results shortly, after we have accounted for the interpretations of or from the point of view of linguistic-pragmatics.

Here is a brief account of how implicatures come about according to what is often referred to as a "neo-Gricean" family of approaches (Gazdar, 1979; Horn, 1973; for a review, see Levinson, 1983). The connectives or and and may be viewed as part of a scale $(<$ or, and $>$ ), where and constitutes the more informative element of the scale (as $p$ and $q$ entails $p$ or $q$ ). In the event that a speaker chooses to utter a disjunctive sentence, $p$ or $q$, the hearer will take it as suggesting that the speaker either has no evidence that a stronger element in the scale, i.e., $p$ and $q$, holds or that he or she perhaps has evidence that it does not hold. Thus, presuming that the speaker is cooperative and well informed, the hearer will tend to infer that it is not the case that $p$ and $q$ both hold, thereby interpreting the disjunction as exclusive. According to this approach, this mechanism is fully general and applies whenever we have lexical items that can be generally construed as part of an informativeness scale. The novel contribution of this approach is its notion of scales, often known as Horn scales, which are obviously critical for ultimately drawing an implicature.

Another account comes from Relevance Theory (Sperber \& Wilson, 1986/ 1995). In the Relevance framework, an implicature is defined as an inference that the speaker intends and expects the hearer to draw in order to arrive at an interpretation of the utterance that is relevant enough. Relevance Theorists do not disagree with the mechanics of the scalar implicature but with the explanation for when it arises. According to Relevance Theory, a scalar implicature is derived when a relatively weak statement fails to meet the hearer's expectation of relevance. These attempts are governed by principles concerning effect and effort; namely, listeners try to gain as many effects as possible for the least effort.

\footnotetext{
${ }^{2}$ This by no means exhausts the literature on disjunctions. For example, after Evans and Newstead (1981) had investigated matching bias (the non-logical preference to construct cases whose components match rather than mismatch the named values in a rule) in conditionals, they investigated disjunctions to determine whether or not this bias was generalisable to other connectives. Other investigations concerned the negation's (obfuscating) role in disjunctive processing (Evans \& Newstead, 1980; Roberge, 1974, 1976a), the role of materials in disjunctive comprehension (Noveck \& Politzer, 1998; Van Duyne, 1974), and the role of disjunction presentation (i.e., as inclusive vs exclusive [Roberge, 1976b] or as exclusive vs unspecified [Noveck \& Politzer, 1998]). More recently, one finds disjunctions being used opposite conditionals in Mental Model investigations of cognitive illusions (Johnson-Laird, Legrenzi, Girotto, \& Legrenzi, 2000), or as a source of modal reasoning (Bell \& Johnson-Laird, 1998). Overall, one can conclude that (a) matching bias is not generalisable to disjunctions; (b) disjunctions are processed more easily when they are presented exclusively rather than inclusively; and (c) materials matter to the extent that laboratory tasks that use letters (There is a P or a Q) are easier to process than those that use people in cities (Monica is in Paris or Noemi is in Lyon). None of these findings is highly relevant to our present aims, however, which is to uncover the factors that predict each of the two interpretations of $o r$.
} 
If the situation warrants the effort, people will draw out the scalar implicature, which is an effect.

One difference between the two approaches concerns the degree of automaticity with which implicatures take place. The neo-Gricean account assumes that while the inclusive interpretation is the more basic one (because the exclusive one is derived as an addition or a further specification of the former), the exclusive interpretation is actually the default in simple unembedded contexts (this is a crucial proviso-see footnote 1). This simply reflects the observation that in such situations the exclusive interpretation is stronger, i.e., more informative, than the inclusive one; hence, unless there is something in the context of the utterance that explicitly blocks it, the hearer will tend to assume that such an interpretation is indeed the intended one. Relevance theory does not assume that the implicature is the default in this sense, but that it gets produced when searching for a relevant interpretation of an utterance. However substantive this difference may turn out to be, we will not get any further into this debate and we will feel free to adopt teminology drawn from any of the approaches ultimately inspired by Grice to describe the phenomena that concern us (be it neo-Gricean or relevance-theoretic terminology). What is important for our purposes is that the basic interpretation of $o r$ is inclusive and that the exclusive interpretation can be shown to arise in certain classes of situations.

\section{ARRIVING AT EXCLUSIVE INTERPRETATIONS}

The two approaches agree on one situation where one should find such an implicature - when or underspecifies an anticipated conjunctive assertion. That is, if a conjunctive conclusion ( $p$ and $q$ ) is implied but a mere disjunction ( $p$ or $q$ ) is heard, the listener will tend to adopt that not $p$ and $q$ holds because this implicature-driven interpretation of or is more informative. Consider Tony's disjunctive reply in the following exchange, how it underdetermines George's expectations, and thus implies not both:

(2) George: So, Chirac and Jospin are coming for dinner then.

Tony: Well, we'll have Chirac or Jospin.

By considering the set of situations that can be accounted for by both sorts of disjunction, one can see how the exclusive interpretation of Tony's statement in (2) is more informative (accounts for a narrower set of circumstances) than an inclusive interpretation; i.e., whereas an exclusive interpretation can account for two situations (1. Chirac coming without Jospin; 2. Jospin coming without Chirac), an inclusive interpretation would allow for a third (3. both Chirac and Jospin coming). Thus, assuming that George is looking for an informative reply, he will draw the implicature and arrive at an exclusive interpretation. Relevance theorists would argue that the contrast between Tony's disjunction with George's prior conjunction prompts an effort that yields an effect, an exclusive 
interpretation by way of the implicature. On the neo-Gricean approach this is an example of the general case in which a disjunctive statement is picked over a stronger conjunctive alternative. As the speaker avoids the stronger alternative (Tony does not simply answer "yes" to George's appeal for confirmation), this will be taken as evidence against or's conjunctive interpretation, thereby reinforcing the tendency to an exclusive construal.

The example in (2) also highlights the difference between disjunctive statements used in conversation and those used in the studies that reveal a tendency towards inclusive interpretations (Braine \& Rumain, 1981; Evans \& Newstead, 1980; Paris, 1973). The scenario in the example, whose disjunction we assume is clearly exclusive, is arguably analogous to the reasoning scenarios in the studies in which the exclusive interpretation does not appear to be obvious to participants (the scenarios in each of the three studies anticipates a conjunction - e.g., a boy riding a bicycle and a dog lying down; a P and a 4; a cat and a frog-but presents a disjunction instead). This leads to the following question: Why does one find low rates of exclusive interpretations in such experimental scenarios?

We argue that the likelihood of drawing scalar implicatures is linked to the relative difficulty of the conversational context; formal reasoning tasks, being relatively artificial, depress rates of implicature production. The differences among the existing findings support this view. Paris's study, which is arguably the most challenging because it requires participants to integrate complex images concerning unrelated events, prompted the highest rates of inclusive interpretations while Braine and Rumain's study, arguably the least challenging, produced the lowest amounts. This implies that the more difficult the problem, (1) the less likely it is that one can produce the implicature, and (2) the more likely one will accept the basic inclusive interpretation.

Developmental evidence supports this analysis as well. In both Paris's (1973) and Braine and Rumain's (1981) studies, young reasoners reveal a preference for inclusive interpretations more than adults. Assuming that adults have more resources than children in an effort to interpret or statements, a reasonable outcome would be that children accept the basic inclusive interpretation and that adults go further by producing the scalar implicature. Noveck (2001) provides supporting evidence for this developmental claim with other weak scalar terms like might and some, which developmentally behave like or. For example, he showed that children are less likely than adults to produce the implicature Not All from Some.

These claims about relative difficulty are relevant here because we do not suppose that there is anything intrinsic about the materials employed in the three prior studies that prevents exclusive interpretations. We also do not assume that there is some kind of exclusiveness effect based solely on content. The materials we will be employing are arbitrary in nature but maximally simple enough to allow for the production of implicatures and exclusive interpretations. 


\section{ARRIVING AT INCLUSIVE INTERPRETATIONS}

Another related endeavour is to uncover structural factors that favour inclusive interpretations over exclusive interpretations. Are there classes of contexts in which, say, an inclusive interpretation of or remains unperturbed? The answer appears to be affirmative. In this respect, too, the linguistic-pragmatic literature has proven to be a useful guide. ${ }^{3}$ Drawing freely from such literature (and in particular from Chierchia, 2001), the generalisation that seems to be emerging is the following:

(3) Or is interpreted inclusively in contexts in which any is licensed.

The English determiner any has two main uses. On the one hand it is a Negative Polarity Item and requires "negative" contexts. On the other it is a Free Choice Item (akin to German irgendein or Italian qualunque) and requires certain kind of modalities. These uses are illustrated in what follows.

(4) a. There aren't any cookies in the jar.

b. ${ }^{*}$ There are any cookies in the jar. ${ }^{4}$

Another exemplary context that appears to license elements like any are the antecedents of conditionals:

(5) a. If there are any cookies left, we are in luck.

b. * If we are in luck, there are any cookies left.

Also important is the case of questions and optative operators:

(6) a. Are there any cookies left?

b. Applicants should be familiar with any major word processing program.

Example (6b), in particular, illustrates the so called "free choice" use of any. Interestingly, the contexts described in (5) and (6) are the same ones in which an inclusive interpretation of or appears to emerge readily:

(7) a. If Jules or Jim come, the party will be a success.

b. Do you have a pencil or a pen?

c. Applicants should have a degree or experience in computing.

${ }^{3}$ Discussion of these matters can be found in Geis and Zwicky (1971), Gazdar (1979), Horn (1989, pp. 233 ff.), and Fauconnier (1975). For a more recent discussion, see Chierchia (2001).

${ }^{4}$ Note that we adopt the linguistic convention of denoting non-grammatical sentences with an asterisk. 
With respect to (7a), if both Jules and Jim come, then the party will be considered a success; the antecedent's truth conditions are fulfilled. Similarly, if one has both a pencil and a pen, one would answer "yes" to question (7b), suggesting that or is being construed inclusively. With respect to sentences like (7c), there is empirical support (Newstead, Griggs, \& Chrostowski, 1984) showing that an inclusive interpretation of or emerges. Newstead et al. found that a "qualification" disjunction like the one in (7c) is likely to lead to an inclusive interpretation, unlike other comparable disjunctive statements (involving threats, promises, etc.). Part of what we plan to do with our experiments is provide support for the other two inclusive construals of or in (7), namely with sentences like (7a) and (7b).

What do the contexts in (4)-(7) have in common? We think that best answer to this question remains the one put forth in Ladusaw's (1979) seminal work; namely, the relevant contexts are downward-entailing. Downward entailment is a semantic property of contexts in which we can draw an inference from sets to their subsets (cf. Ladusaw, 1979). The prime and prototypical example is constituted by "negative" contexts. For example, consider the statements in (8a) and ( $8 b)$ and the statements that they readily entail, respectively, in (9a) and (9b).

(8) a. John did not buy ice cream.

b. John's tie is not red.

(9) a. John did not buy chocolate ice cream.

b. John's tie is not bright red.

If we claim that John does not belong to the set of those that bought ice cream, we also thereby say he does not belong to the set of those that bought chocolate ice cream. This illustrates in what sense negation constitutes a downward-entailing context, i.e., a context where inferences from sets to subsets are licensed.

By considering negated cases of or and the implied subsets they entail, one can see why disjunction is inclusive in such contexts. Consider DeMorgan's law and how the sentence John did not buy ice cream or chips is logically equivalent to John did not buy ice cream and he did not buy chips. If the disjunction were exclusive, John did not buy ice cream or chips would be considered consistent with the situation where he bought both ice cream and chips. The fact that inclusive interpretations are preferred in negative contexts is consistent with the informativeness-based account described earlier, because, in these negative cases, inclusive interpretations make for a more restricted set of possibilities than exclusive ones. That is, an inclusive interpretation in John did not buy ice cream or chips allows for one possibility (1. he bought neither ice cream nor chips) whereas an exclusive interpretation would lead to two (1. he boughtneither 2. he bought both). Thus, in negative contexts the inclusive interpretation is actually stronger (more informative) than the exclusive one. In other words, if we have a 
scale $<$ or, and $>$, in non-downward-entailing contexts, and entails or. Negation, and downward-entailing contexts in general, reverse this state of affairs, making (inclusive) or the strongest pole. Which explains why the classical exclusiveness implicature need not arise in such contexts. If a context is downward-entailing, an inclusive construal yields a stronger (i.e., more informative) statement and so the exclusiveness implicature is not in a position to arise.

The same holds, mutatis mutandis, for the antecedents of conditionals. The truth of the utterance If Jules or Jim come, then the party is a success with an inclusive interpretation of the disjunction is more informative than (i.e., entails) the same utterance with an exclusive interpretation of the disjunction. That is, there are fewer cases where the utterance is true (or more cases that are false) when the disjunction is inclusive rather exclusive. It is just as with negation. ${ }^{5}$

Taking stock, the distribution of cases revealing an inclusive interpretation of or appears to overlap with the distribution of cases that license any. This suggests that the contexts that favour inclusive construals indeed form a linguistically natural (and hence structurally determined) class, as grammar treats them alike $v i s-\grave{a}$-vis a seemingly unrelated phenomenon, namely the distribution of any. Moreover, the latter has been observed to correlate with downwardentailingness, and such an insight paves the way to understanding the interpretation of or in terms of a rather simple, information-based account: we tend not to draw implicatures when that would weaken what we say. We now turn to our attempt at providing experimental support for this idea.

\section{THE EXPERIMENTS}

In what follows, we provide experimental evidence in favour of the view that the linguistic factors described earlier, such as implicatures and downwardentailingness, affect the interpretation of $o r$. Our main goal is at least twofold. First, we want to show that such phenomena play a role in reasoning tasks. Second, by using maximally simple arbitrary materials, letters written on a hidden blackboard (which are arguably less cumbersome than those in the previous studies cited), we hope to demonstrate how linguistic properties of the sentences can affect the interpretation of $o r$ without referring to specific kinds of realistic content (or more generally to scripts or schemas).

Experiment 1 investigates the implicature related to disjunctions. We create a simple problem whose features favour the production of the implicature. It includes a premise set that implies a conjunctive conclusion $(P$ and $Q)$ but offers

\footnotetext{
${ }^{5}$ Although most any-licensing contexts are clearly downward-entailing, it is unclear whether they all are. It is, for example, controversial whether questions or imperatives can readily be regarded as downward-entailing. One of Chierchia's (2001) points is that even if downwardentailingness will ultimately be replaced by some more general characterisation of the relevant contexts, the empirical correlation between any-licensing and inclusive construals appears to stand.
} 
a disjunctive conclusion ( $P$ or $Q$ ) instead. The expectation is that the implicature for or will be triggered (not both $P$ and $Q$ ), leaving the disjunction prone to an exclusive interpretation. Experiment 1 also investigates a downward-entailing context that ought to prompt an inclusive interpretation-antecedents of conditionals - to test the claim that or is interpreted inclusively in such situations.

Experiments $2 \mathrm{~A}$ and $2 \mathrm{~B}$ address an alternative account of our claims concerning the anticipated exclusive interpretation for the first of the Implicature problems described earlier, which is that participants reject the provided conclusion $P$ or $Q$ in Experiment 1 (when $P$ and $Q$ is implied) solely on grounds of an independent kind of pragmatic infelicity. That is, perhaps participants assume an inclusive interpretation of or and a rejection of the provided conclusion is due to its being underinformative. In Experiments $2 \mathrm{~A}$ and $2 \mathrm{~B}$, we (a) determine whether or not this account is tenable, and (b) provide additional reasoning problems in which we expect or to be construed exclusively. With respect to (a) we present an additional problem containing the premise set If $P$ then $Q$ and $R ; P$ and the conclusion $Q$. A rejection of this provided conclusion would be supportive of the independent infelicity account whereas an affirmation would appear to constitute evidence against it. With respect to (b), Experiment $2 \mathrm{~A}$, for example, includes a new problem, If $P$ then $Q$ or $R ; P / / N$ ot both $Q$ and $R$; an affirmative response to this problem would appear to provide supplementary support to our claim that or in such cases is treated exclusively.

Both Experiments 3 and 4 investigate question forms and the expectation that they provide a condition for an inclusive interpretation. In Experiment 3, we make one minor modification to Experiment 1 - the provided conclusions are offered as questions. The expectation is that $P$ or $Q$ in a question format (Is there is a $P$ or a $Q$ ?) allows for an inclusive interpretation. Thus, participants ought to respond affirmatively when this question is posed with respect to an implied conjunctive outcome $(P$ and $Q)$. In Experiment 4, we present both forms of the task (from Experiment 1 and Experiment 3 ) to the same participants but in one of two orders so that we can (a) provide support for the phenomena within one overarching experiment and (b) determine the extent to which a participant's first response to a Implicature-Potential problem can influence interpretations to the second.

\section{EXPERIMENT 1}

We establish the conditions in which participants are expected to draw out an implicature. Concretely, imagine a problem whose premises are If $P$ then $Q$ and $R$ and $P$ and whose conclusion is $Q$ or $R$. One would expect participants to be dissatisfied with this conclusion, even though it is valid (with an inclusive interpretation of or). According to linguistic pragmatic theory, the conclusion $Q$ or $R$ with or interpreted inclusively is not as informative or as relevant as 
expected. The implicature (but not both) will be added to the disjunction, making the disjunction exclusive, and will justify a negative response. We provide such a problem and call it the Implicature-Potential problem.

In contrast, when a disjunction is in the antecedent of a conditional, setting up a Disjunctive Modus Ponens problem, the expectation is that or will be treated inclusively. If a reasoning problem includes the premises If $P$ or $Q$ then $R$ and $P$ and $Q$, one would expect that participants would accept the provided conclusion, $R$. If the disjunction were considered exclusive, then one ought to expect participants to "deny the antecedent" and reject the conclusion, at least to the same extent that participants do on standard conditional problems. The Disjunctive Modus Ponens problem presented conjunctions in two ways: In one problem, the and was explicit in the minor premise (There is a $P$ and a $Q$ ); in the other problem, the conjunction was implicit (two premises were presented as There is a $P$; There is a $Q$ ). Our expectation is that a conjunctive minor premise (or set of minor premises) will be considered compatible with the disjunctive antecedent of the major premise and conditional's consequent will be inferred.

We point out two features of the experimental procedure. One is motivated by the concern that repeated problems can make participants readily aware of the experimenter's intent and can easily affect participants' interpretations. In order to encourage spontaneous reactions, no problem is presented twice. The other feature is that we include numerous auxiliary problems in order to verify that (a) experimental manipulations had their desired effect, and that (b) the expected inclusive interpretations are confined to those situations described earlier. With respect to (a) this means that other problems verify, for example, that the implicitly conjunctive minor premises (There is a $P$; There is a $Q$ ) are indeed treated conjunctively in a Conjunctive Modus Ponens problem. With respect to (b) this means it is important to show, not only that we get a "hit" (evidence of an inclusive interpretation) when conditions are favourable, but that we do not get a "hit" when conditions are not. For example, it is important to demonstrate that participants view and and or as incompatible when and is in the antecedent and or in the minor premise (as in If $P$ and $Q$ then $R$; $P$ or $Q$ ). The premises are not expected to lead to the endorsement of $R$ because the or in this case will not be considered sufficient for prompting the conjunctive modus ponens inference.

\section{Method}

Participants. A total of 20 Masters students from the Institut des Sciences Cognitives and the Université de Grenoble volunteered to participate. Only those who had no background in logic were included. Participants' backgrounds were mostly in fields related to the neurosciences and psychology.

Materials. A total of 18 propositional reasoning problems were prepared. See Tables 2 and 3 for a complete listing of the presented problems. Four had a 
disjunctive conditional as a major premise (If there is a $P$ or a $Q$ then there is an $R$ ). Four had a conjunctive conditional as a major premise (If there is a $P$ and $a$ then there is an $R$ ). Two conditionals had disjuncts as a consequent (If there is a $P$ then there is a $Q$ or an $R$ ) and two had a conjunct as a consequent (If there is a $P$ then there is $a Q$ and an $R$ ). Two employed a disjunction in the first premise and included three premises in total; the two differed in that the conclusion contained a disjunction or else a conjunction. Finally, there were four problems that are often the focus of studies in the reasoning literature (one modus tollens problem, one negated conjunction problem in which the first element is affinned, one negated conjunction problem in which the second element is affirmed, and one that requires a standard disjunction-elimination inference). These were included for two reasons: (1) to verify that the population was comparable to others found in the literature; and (2) to add problems that call for a negative response (otherwise, an especially large majority of problems would require an affirmative response).

The Implicature-Potential Problem (\#1) and the two Disjunctive Modus Ponens problems (\#2 and \#3) in Table 2 are of direct interest to the study. The others serve two purposes. One is that they are intended as controls of one form or another. Problems 4 and 5 are verifications that participants carry out a Disjunctive Modus Ponens problem when there is either one disjunct or two in the minor premise. Problems 6-9 verify that two conjuncts are needed when two are called for and that one conjunct — or a disjunction — is not enough for carrying out a Conjunctive Modus Ponens problem. Problem 10 was included to verify that participants carry out Modus Ponens problems that have disjunctions in their consequents. Problem 11 was included to verify that participants carry out Modus Ponens problems that have conjunctions in their consequents. Problem 12 was included to verify that a provided conjunctive conclusion does not satisfy the conditions for Modus Ponens problems having or in the consequent (unlike Problem 3 of Table 1 which has or in the antecedent and and in the minor premise). That is, an or in the consequent does not operate like or in the antecedent. Problems 13 and 14 added a little extra work (three premises instead of two), but are added to verify the results of Problems 10 and 12. The remaining problems were the four problems often investigated in the propositional reasoning literature. The other purpose for the many control problems is to have filler items. Having a variety of filler items and one instance of each experimental problem among them takes the focus off the main experimental problems, hides the task's real intentions, and ensures that participants' responses are spontaneous.

Although all these examples use P, Q, R etc., the test problems used all the letters in the alphabet that are pronounceable in one syllable in French (which excludes $\mathrm{W}$ and $\mathrm{Y}$ ). No letter was used disproportionately more than the others. Also, letter combinations that could be construed to have meaning were avoided. 
Design and procedure. The experiment was presented on a portable Macintosh computer with Psyscope software (Cohen, MacWhinney, Flatt, \& Provost, 1993). The participants were instructed to carefully read statements concerning the presence or absence of certain letters on a hidden blackboard. The instructions further explained that the statements are presented as premises and that after each group of premises there is a conclusion to be evaluated as correct, not correct or "Not enough information to decide". The three options were indicated on the appropriate keys on the keyboard ("No" was on the upper left portion of the keyboard, the "Insufficent Information" option was in the upper middle portion, and the "Yes" option was on the upper right). They were further told to read the text naturally and that the task was not complicated. Finally they were asked to rest their two hands in such a manner that their thumbs were on the space bar, one index finger was on the "Yes" key, and that the other was on the "No". This was followed by two examples. One was a transitive condition problem (translated from French):

If there is an $\mathrm{M}$ then there is an $\mathrm{H}$.

If there is a $\mathrm{W}$ then there is an $\mathrm{M}$.

There is a W.

There is an $\mathrm{H}$.

and the other required modus ponens with a negated consequent:

If there is a B then there is not an $\mathrm{E}$.

There is a $\mathrm{B}$.

There is an E.

The computer program then presented the 18 test problems in a random order.

\section{Results}

The principal results are presented in Tables 2 and 3. We first consider performance with respect to standard reasoning problems before investigating the three problems in Table 2 that are most relevant to our study. We then analyse these three problems with respect to chance predictions and, where useful, to each other. Finally, we take a look at the remaining problems from Table 3 in order to see how they shed light on our main findings.

Rates of correct responses to the four commonly studied problems in the literature (modus tollens, the two kinds of negated conjunction problems, the disjunction elimination problem) appear to fall within the norms of similar problems in the literature. Rates of correct responses to modus tollens are rather 
Experiment 1: Problems 1, 2, and 3

\begin{tabular}{|c|c|c|c|c|c|}
\hline & Premises & Conclusion & Yes & No & II \\
\hline 1. & $\begin{array}{l}\text { If there is a } P \text { then } \\
\text { there is a } Q \text { and an } R \text {. } \\
\text { There is a } P \text {. }\end{array}$ & There is a $\mathrm{Q}$ or an $\mathrm{R}$. & 25 & $75^{*}$ & 0 \\
\hline 2. & $\begin{array}{l}\text { If there is a } \mathrm{P} \text { or a } \mathrm{Q} \\
\text { then there is an } \mathrm{R} \text {. } \\
\text { There is a } \mathrm{P} \text {. } \\
\text { There is a } \mathrm{Q} \text {. }\end{array}$ & There is an R. & $85^{*}$ & 15 & 0 \\
\hline 3. & $\begin{array}{l}\text { If there is a } \mathrm{P} \text { or a } \mathrm{Q} \\
\text { then there is an } \mathrm{R} \text {. } \\
\text { There is a } \mathrm{P} \text { and a } \mathrm{Q} \text {. }\end{array}$ & There is an R. & 60 & 40 & 0 \\
\hline
\end{tabular}

Percentage of participants responding "Yes", "No", or "Insufficient Information to decide (II)" to the Implicature-Potential problem (\#1), the Disjunctive Modus Ponens problem with implicitly conjunctive minor premises (\#2), and the Disjunctive Modus Ponens problem with an explicitly conjunctive minor premise (\#3) in Experiment 1. $N=20$.

$* p<.05$.

high, $80 \%$, but this is not an exceptional finding. Thus, the population appears to be like others in the literature.

Taking a conservative tack (i.e., ignoring the Insufficent Information option) for problems having 20 participants, a given type of response is at a rate significantly above chance levels when a cell reveals that 15 (75\% of) participants answer similarly. With this standard, which will be applied throughout the paper, one can conclude that a significant number of participants (15) employed an exclusive interpretation (i.e., drew the implicature) by saying "no" to the Implicature-Potential Problem (If $P$ then $Q$ and $R ; P / / Q$ or $R$ ).

Similarly, a significant percentage (85\%) agree that the minor premises There is a $P$; There is a $Q$; satisfy conditions for inferring the consequent in the disjunctive modus ponens problem (Problem \#2 in Table 2) and apparently treat the disjunction inclusively. Rates of inclusive interpretations to problem If $P$ or $Q$ then $R ; P$ and $Q / / R$ yielded rates of correct responses $(60 \%)$ that only approach significance. However, by treating the two latter disjunctive Modus Ponens problems together and by comparing the outcomes to chance, rates of agreement (indicating an inclusive interpretation) are significantly greater than predictions based on chance, $\chi^{2}(2)=9.9, p<.01 .^{6}$ Nearly all (11 of 12$)$ participants who

${ }^{6}$ Participants are independently distributed among three categories. That is, a participant agrees with the provided conclusion on (a) both problems, (b) one of two problems, or (c) neither of the two problems. The outcomes for these are compared to chance where category (b) is likely to occur $50 \%$ of the time and categories (a) and (c) $25 \%$ each. 
Experiment 1: Problems 4-18

\begin{tabular}{|c|c|c|c|c|c|}
\hline & Premises & Conclusion & Yes & No & $I I$ \\
\hline 4. & $\begin{array}{l}\text { If } P \text { or } Q \text { then } R \text {. } \\
P \text {. }\end{array}$ & $\mathrm{R}$. & 95 & 5 & 0 \\
\hline 5. & $\begin{array}{l}\text { If } P \text { or } Q \text { then } R \text {. } \\
P \text { or } Q \text {. }\end{array}$ & $\mathrm{R}$. & 90 & 10 & 0 \\
\hline 6. & $\begin{array}{l}\text { If } P \text { and } Q \text { then } R \text {. } \\
P \text { and } Q \text {. }\end{array}$ & $\mathrm{R}$. & 100 & 0 & 0 \\
\hline 7. & $\begin{array}{l}\text { If } P \text { and } Q \text { then } R \text {. } \\
\text { P. } \\
\text { Q. }\end{array}$ & $\mathrm{R}$. & 95 & 5 & 0 \\
\hline 8. & $\begin{array}{l}\text { If } P \text { and } Q \text { then } R \text {. } \\
Q \text {. }\end{array}$ & $\mathrm{R}$. & 5 & 75 & 20 \\
\hline 9. & $\begin{array}{l}\text { If } P \text { and } Q \text { then } R \text {. } \\
P \text { or } Q \text {. }\end{array}$ & $\mathrm{R}$. & 20 & 80 & 0 \\
\hline 10. & $\begin{array}{l}\text { If } \mathrm{P} \text { then } \mathrm{Q} \text { or } \mathrm{R} \text {. } \\
\mathrm{P} \text {. }\end{array}$ & Q or R. & 95 & 5 & 0 \\
\hline 11. & $\begin{array}{l}\text { If } P \text { then } Q \text { and } R \text {. } \\
P \text {. }\end{array}$ & $\mathrm{Q}$ and $\mathrm{R}$. & 95 & 5 & 0 \\
\hline 12. & $\begin{array}{l}\text { If } \mathrm{P} \text { then } \mathrm{Q} \text { or } \mathrm{R} \text {. } \\
\mathrm{P} \text {. }\end{array}$ & $\mathrm{Q}$ and $\mathrm{R}$. & 20 & 70 & 10 \\
\hline 13. & $\begin{array}{l}\text { P or Q. } \\
\text { If } \mathrm{P} \text { then } \mathrm{R} \text {. } \\
\text { If } \mathrm{Q} \text { then } \mathrm{S} \text {. }\end{array}$ & R or $\mathrm{S}$. & 80 & 5 & 15 \\
\hline 14. & $\begin{array}{l}P \text { or } Q . \\
\text { If } P \text { then } R . \\
\text { If } Q \text { then } S .\end{array}$ & $\mathrm{R}$ and $\mathrm{S}$. & 10 & 75 & 15 \\
\hline 15. & $\begin{array}{l}\text { If } \mathrm{P} \text { then } \mathrm{Q} \text {. } \\
\text { no } \mathrm{Q} \text {. }\end{array}$ & P. & 10 & 80 & 10 \\
\hline 16. & $\begin{array}{l}\text { P or } Q \text {. } \\
\text { no } Q \text {. }\end{array}$ & P. & 75 & 10 & 15 \\
\hline 17. & $\begin{array}{l}\text { Not both P and Q. } \\
\text { P. }\end{array}$ & $\mathrm{Q}$. & 10 & 90 & 0 \\
\hline 18. & $\begin{array}{l}\text { Not both } \mathrm{P} \text { and } \mathrm{Q} \text {. } \\
\text { Q. }\end{array}$ & P. & 5 & 95 & 0 \\
\hline
\end{tabular}

The remaining problems and their rates of "Yes", "No", and "Insufficient information to decide (II)" responses in Experiment 1. $N=20$.

treated or inclusively in If $P$ or $Q$ then $R ; P$ and $Q / / R$ (Problem \#3) also treated or inclusively in If $P$ or $Q$ then $R ; P ; Q / / R$ (Problem \#2) and just two participants treated or exclusively in both sorts of problems. This means that six participants who treated or inclusively when the conjunction was implicit treated or exclusively when and was employed explicitly in the minor premise. Rates of inclusive responses across Problems 2 and 3 are not significantly different from each other. 
The control problems confirm that the minor premises in the experimental problems had their desired effect. For example, when there are two premises of the sort There is a $P$; There is a $Q$, they satisfy the antecedent of the Conjunctive Modus Ponens problem and the consequent is inferred (see Problem 7) so one can be assured that this subset of premises was interpreted as a conjunction in Problem 2 (If P or $Q$ then $R ; P ; Q / / R$ ). Problem 9 is an interesting control problem because only $20 \%$ of participants treat the minor premise $P$ or $Q$ as compatible with its conditional's antecedent If $P$ and $Q$ then $R$. When Problems 3 and 9 are paired and treated statistically (participants can be categorised based on four independent patterns of response: both Problems 3 and 9 prompt "Yes"; Problem 3 prompts "yes" and Problem 9 "No"; Problem 3 prompts "No" and Problem 9 "Yes"; or both prompt "No"), the results indicate a significant difference, $\chi^{2}(3)=$ $8.8, p<.05$. This shows that or and and are not interchangeable in a conditional's antecedent; a conjunctive minor premise is largely compatible with a disjunctive antecedent whereas a disjunctive minor premise is not compatible with a conjunctive antecedent. The remaining control problems also demonstrate that and is readily distinguishable from $o r$. Whereas one disjunct in (\#4) is enough to infer the consequent, one conjunct in (\#8) is not. Whereas a disjunctive conclusion is considered valid in Problems 10 and 13, a conjunctive conclusion is not in Problems 12 and 14.

\section{Discussion}

This experiment reports two main findings. The first is that participants find that or is not a satisfying connective in a conclusion when a conjunction is called for. When a reasoning problem has the premises If $P$ then $Q$ and $R ; P$, the conclusion $Q$ or $R$ is evaluated negatively. Linguistic-pragmatic theory describes why: interpreted inclusively, the provided conclusion is weaker than the implied conclusion. This prompts an implicature of the sort "but not both", ultimately rendering the disjunctive conclusion exclusive. This finding confirms that when a disjunctive assertion is weaker than is warranted by the manifest evidence, it produces an implicature. Assuming our cognitive effort account is correct, italso implies that our manipulation is easier for participants than comparable ones investigated earlier (Braine \& Rumain, 1981; Evans \& Newstead, 1980; Paris, 1973 ) because our percentage of exclusive interpretations is noticeably higher than in any of these studies.

The second main finding is that disjunctions in the antecedent of a conditional are likely to be interpreted inclusively. Thus, when a disjunctive conditional If $P$ or $Q$ then $R$ is followed by $P$; $Q$ or by $P$ and $Q$, participants are likely to generate the Modus Ponens inference. If or were treated exclusively by default (at least at the rate of exclusiveness reported for the Implicature-Potential Problem), one would expect low rates of affirmative responses. Evidently, the conjunctive minor premise (or set of minor premises) is compatible with the disjunction in the 
conditional's antecedent. This is not the case when the and and $o r$ are reversed. When a conjunctive conditional If $P$ and $Q$ then $R$ is followed by a disjunction $P$ or $Q$, the minor premise is not treated inclusively and is not considered sufficient for inferring $R$. A significant percentage of participants end up committing the denial-of-the-antecedent fallacy.

Interestingly, the explicit use of and in the second premise of If $P$ or $Q$ then $R$; $P$ and $Q$ makes the contrast between or and and more salient than when and is implicit in If $P$ or $Q ; P ; Q$. One way to understand this is that the explicit mention of and forces participants to contrast it with or. It appears then that participants make sense of such a contrast (i.e., they maximise its relevance) by construing or exclusively. Although the effect of the explicit and is notable, it was not significant when compared to the implicit-conjunction version of the problem.

Similar to the Implicature-Potential problem, Problems 12 and 14 show that an exclusive interpretation arises when a disjunctive conclusion is implied and juxtaposed with a presented conjunctive one. This indicates that or in such circumstances is also open to implicature. There are grounds to suppose that participants would make the effort to render it as informative as possible. These problems were included initially as controls, but they could equally qualify as two other Implicature-Potential problems. However, the similarity between the Implicature-Potential problem and the Problems 12 and 14 ends there. As will be seen later, we anticipate that disjunctive conclusions presented in the form of a question are typically treated inclusively and only the original ImplicaturePotential problem provides us with the means to test for this.

\section{EXPERIMENTS 2A AND 2B}

In these Experiments, we consider an alternative explanation of our findings concerning the original Implicature-Potential problem in Experiment 1, which is that participants reject the conclusion $P$ or $Q$ in Experiment 1 (when $P$ and $Q$ is implied) because they sustain an inclusive interpretation throughout and reject the conclusion on grounds of pragmatic infelicity of a different kind (JohnsonLaird, personal communication). That is, participants perhaps do have an inclusive interpretation of $o r$ in the Implicature Problem and the rejection of the provided conclusion is due solely to its being underinformative (and thus not due to the production of the implicature and a concomitant exclusive interpretation). This objection is just, and calls for further investigation.

To investigate generally whether or not underinformativeness is grounds for rejection, we present an additional problem containing the premise set If $P$ then $Q$ and $R ; P$ and the conclusion $Q$. On the one hand, participants ought to endorse this conclusion because it is valid, even if it is trivial. On the other hand, there are two possible reasons why the provided conclusion could be rejected: One is because it underdetermines the implied conclusion. Judging from the premise set of one of the control problems (If $P$ and $Q$ then $R$; $P$ ), in which most participants 
committed the fallacy of Denying the Antecedent, there is reason to assume that participants will consider a conclusion like $Q$ underdeterminative when $P$ and $Q$ is implied. Thus, if participants provide an affirmative response, there would be reason to doubt that underinformativeness is sufficient grounds for rejecting the conclusion. The second potential cause for negative responses is that, when $Q$ and $R$ is implied, the provided conclusion $(Q)$ might prompt an implicature of the sort $Q$ and not $R$. Although there are no lexical scales that link $Q$ and $R$ with $Q$, there is an entailment scale. Perhaps the entailment scale is enough to provoke an implicature. In sum, there are several reasons to predict a negative response to If $P$ then $Q$ and $R ; P / / Q$, including underinformativeness. An affirmative response would at the least lend doubt to the hypothesis that infelicity alone is sufficient for a negative response. This problem appears in both Experiments $2 \mathrm{~A}$ and $2 \mathrm{~B}$.

There are two experiments described here because we also introduce two new problems that we predict ought to lead to exclusive construals. In Experiment $2 \mathrm{~A}$, we take advantage of findings from Problem 12 of Experiment 1 (If $P$ then $Q$ or $R ; P / / Q$ and $R$ ) which showed that $75 \%$ of participants rejected the conclusion. Our analyses would have to claim that the implied disjunctive conclusion was interpreted as exclusive. In Experiment $2 \mathrm{~A}$, we include a new problem that has the same premise set as Problem 12's (If $P$ then $Q$ or $R ; P$ ) while presenting a negated conjunction (not both $Q$ and $R$ ) as a conclusion. If participants view the premises' implied conclusion $(Q$ or $R$ ) as an inclusive disjunction, then the provided conclusion (not both $Q$ and $R$ ) ought to be readily considered false because an inclusive interpretation of the implied conclusion contradicts the provided conclusion. Thus, an affirmative response to this new problem arguably arises only because the implied conclusion yields an implicature (thus an exclusive interpretation).

In Experiment 2b, we present another similar problem in which the implied conclusion is not both $P$ and $Q$ and the provided conclusion is the disjunction $P$ or $Q$. If the provided conclusion is readily interpreted as an exclusive disjunction, as the Implicature Problem in Experiment 1 appears to indicate, then this problem too ought to prompt an affirmative response. If participants treat the provided disjunctive conclusion inclusively then one ought to find rejections because an inclusive interpretation of or contradicts a negated conjunction. Thus, this problem is relevant because an affirmative response would provide further evidence that a conclusion like $P$ or $Q$ is treated as exclusive in these tasks.

\section{Experiment 2A: Method}

Participants. A total of 25 undergraduate students from the Université de Lyon volunteered to participate. Only those who had no background in logic were included. Participants' backgrounds were mostly in fields related to the social sciences. 
Materials, design and procedure. This experiment was identical to Experiment 1, with the sole exception concerning three problems that replaced three among the original eighteen. The Implicature Potential problem (\#1 from Table 2) was replaced by If $P$ then $Q$ or $R ; P / /$ not both $Q$ and $R$. A second control problem, If $P$ then not both $P$ and $Q ; P / /$ not both $P$ and $Q$, replaced Problem 11 from Experiment 1 in order to verify that participants are not troubled by giving an affirmative response to a negatively formed conclusion. Finally, the problem If $P$ then $Q$ and $R ; P / / Q$ replaced Problem 18.

\section{Experiment 2A: Results and discussion}

As in Experiment 1, participants' responses to the standard problems are similar to those in the literature and to those in Experiment 1:76\% reject the conclusion in the Modus Tollens problem (\#15), 72\% affirm the conclusion in the disjunction-elimination problem (\#16), and $84 \%$ reject the conclusion in the remaining Negated-Conjunction problem (\#17). Otherwise, the problems in Experiment 2a were largely similar to their counterparts in Experiment 1, with the exception of two problems. The Disjunctive Modus Ponens problem with a conjunctive minor premise (If $P$ or $Q$ then $R$; $P$ and $Q / / R$ ) prompted $88 \%$ of participants to respond affirmatively, which is significantly higher than in Experiment $1, \chi^{2}(2)=7.07, p<.05$. Also, the Problem If $P$ and $Q$ then $R ; Q / / R$

TABLE 4

Experiment 2A

\begin{tabular}{|c|c|c|c|c|c|}
\hline \multicolumn{2}{|c|}{ Premises } & \multirow{2}{*}{$\begin{array}{l}\text { Conclusion } \\
\text { There is a Q. }\end{array}$} & \multirow{2}{*}{$\frac{\text { Yes }}{92 *}$} & \multirow{2}{*}{$\frac{N o}{8}$} & \multirow{2}{*}{$\frac{I I}{10}$} \\
\hline 1 & $\begin{array}{l}\text { If there is a } \mathrm{P} \text { then } \\
\text { there is a } \mathrm{Q} \text { and an } \mathrm{R} \text {. } \\
\text { There is a P. }\end{array}$ & & & & \\
\hline 2 & $\begin{array}{l}\text { If there is a P then } \\
\text { there is a } Q \text { or an } R \text {. } \\
\text { There is a P. }\end{array}$ & $\begin{array}{l}\text { There is not both a Q } \\
\text { and an R. }\end{array}$ & $80 *$ & 16 & 4 \\
\hline 3 & $\begin{array}{l}\text { If there is a } P \text { then } \\
\text { there is not both a } Q \\
\text { and an R. } \\
\text { There is a P. }\end{array}$ & $\begin{array}{l}\text { There is not both a Q } \\
\text { and an R. }\end{array}$ & $92 *$ & 8 & 0 \\
\hline
\end{tabular}

Percentage of participants responding "Yes, "No", or "Insufficient Information to decide (II)" to three new problems in Experiment 2A. These replaced Problem 1 (from Table 2), and Problems 11 and 18 (from Table 3). $N=25$. $* p<.05$. 
led to significantly more affirmative responses (36\%) here than in Experiment 1, $\chi^{2}(2)=6.21, p<.05$.

We focus on the problems that are original to this experiment. As can be seen in Table 4, the problem If $P$ then $Q$ and $R ; P / / Q$, led a significant proportion of participants $(92 \%)$ to respond affirmatively. The results from this problem indicate that infelicitousness is not a sufficient cause for rejecting a provided conclusion, because $Q$ in this case underdetermines the implied conclusion.

Participants answer affirmatively to the problem If $P$ then $Q$ or $R ; P / /$ Not both $Q$ and $R$ at rates that are significantly above those predicted by chance $(80 \%)$, supporting our hypothesis on two counts: (1) the affirmative response indicates that the disjunction in the consequent is treated as exclusive; if it had been treated as inclusive, it ought to have prompted negative responses (see Problem 12, which yielded negative responses among $80 \%$ of participants here); (2) once one assumes that the disjunction is exclusive because it was brought about by a scalar implicature, it becomes more obvious why participants choose to provide an affirmative response - the implicature matches the proposed conclusion. ${ }^{7} \mathrm{We}$ also found that a new control problem with a negatively formed conclusion, If $P$ then not both $Q$ and $R ; P / / N o t$ both $Q$ and $R$, presented no difficulties for participants, as one can see that a significant proportion (92\%) answered affirmatively.

\section{Experiment 2B: Method}

Participants. A total of 20 undergraduate students from the Université de Lyon volunteered to participate. Only those who had no background in logic were included. Participants' backgrounds were mostly in fields related to the social sciences.

Materials, design and procedure. This experiment was identical to Experiment 1, except that three new problems replaced the three that had been newly included in Experiment 2A. Experiment 1's Implicature-Potential problem (Problem \#1 from Table 2) was replaced by If $P$ then not both $Q$ and $R ; P / / Q$ or $R$. A second control problem, If $P$ then not both $Q$ and $R ; P / / Q$ and $R$, replaced Problem 11 and was included in order to verify that participants can properly reject an implied negated conjunction. Finally, we kept the problem If $P$ then $Q$ and $R ; P / / Q$ which had replaced Problem 18 of Experiment 1.

\footnotetext{
${ }^{7}$ This result also shows why it is hard to argue that infelicitousness alone is a sufficient cause for rejecting a provided conclusion, because even here the provided conclusion underdetermines the implied (exclusive disjunctive) conclusion and yet a significant majority of participants still respond affirmatively.
} 


\section{Experiment 2B: Results and discussion}

As in Experiment 1, participants' responses to the standard problems are similar to those in the literature and to those in Experiment 1: 85\% reject the conclusion in the Modus Tollens problem (\#15), 75\% affirm the conclusion in the disjunction-elimination problem (\#16), and $90 \%$ reject the conclusion in the Negated Conjunction problem (\#17). Otherwise, the problems in Experiment 2B were statistically indistinguishable from their counterparts in Experiment 1, with no exceptions.

We now focus on the problems that are central to this experiment. As can be seen in Table 5, a significant proportion of participants $(75 \%)$ affirm the conclusion in If $P$ then not both $Q$ and $R ; P / / Q$ or $R$. Thus, it appears that the conclusion is being treated as an exclusive disjunction here, just as we claim it is in the Implicature-Potential Problem in Experiment 1. If participants had treated the provided conclusion as an inclusive disjunction, then we should have found a higher rate of rejections, as one does in the new control problem. The new control problem, If $P$ then not both $Q$ and $R ; P / / Q$ and $R$ shows that $90 \%$ of participants reject its conjunctive conclusion. Finally, as in Experiment $2 \mathrm{~A}$, the problem, If $P$ then $Q$ and $R ; P / / Q$, led a significant proportion of participants $(75 \%)$ to respond affirmatively.

TABLE 5

Experiment 2B

\begin{tabular}{|c|c|c|c|c|c|}
\hline & Premises & Conclusion & Yes & No & $I I$ \\
\hline 1 & $\begin{array}{l}\text { If there is a } P \text { then } \\
\text { there is a } Q \text { and an } R \text {. } \\
\text { There is a P. }\end{array}$ & There is a Q. & $75 *$ & 25 & 10 \\
\hline 2 & $\begin{array}{l}\text { If there is a } P \text { then } \\
\text { there is not both a } Q \\
\text { and an R. } \\
\text { There is a } P \text {. }\end{array}$ & There is a $\mathrm{Q}$ or an $\mathrm{R}$. & $75 *$ & 20 & 5 \\
\hline 3 & $\begin{array}{l}\text { If there is a P then } \\
\text { there is not both a } Q \\
\text { and an R. } \\
\text { There is a P. }\end{array}$ & There is a $\mathrm{Q}$ and an $\mathrm{R}$. & 10 & $90 *$ & 0 \\
\hline
\end{tabular}

Percentage of participants responding "Yes, "No", or "Insufficient Information to decide (II)" to three new problems in Experiment 2B. These replaced Problem 1 (from Table 2), and Problems 11 and 18 (from Table 3). $N=20$. $* p<.05$. 


\section{Discussion of Experiments 2A and 2B}

Based on these experiments, it appears unlikely that (a) participants reject the provided conclusion in the Implicature-Potential Problem in Experiment 1 because of an independent kind of pragmatic infelicity, and that (b) they treat the disjunctive conclusion as inclusive. The problem, If $P$ then $Q$ and $R ; P / / Q$, led a significant proportion of participants in both experiments to respond affirmatively, indicating that a conclusion's underinformativeness is not necessarily a cause for rejection. When a premise sets' implied conclusion is a disjunction $(Q$ or $R)$, it is treated as exclusive, as can be seen by the proportion of participants who find the negated conjunction (not both $Q$ and $R$ ) an acceptable conclusion (Experiment 2A). When a disjunction $(Q$ or $R)$ is offered as a conclusion in a problem whose premise set implies a negated conjunction (Not both $Q$ and $R$ ), one finds that a significant proportion affirm the provided conclusion (Experiment 2B). An inclusive interpretation of such a disjunctive conclusion would have led participants to reject it.

\section{EXPERIMENT 3}

As described in the Introduction, question forms are another grammatical context in which or ought to be treated inclusively. This is why this experiment is identical to the first, except that all the conclusions are expressed as questions. Whereas Experiment 1 presented conclusions through assertions, e.g., There is a $Q$ or an $R$, Experiment 3 presents its conclusions by way of questions, as in $I s$ there a $Q$ or an $R$ ? (in French, $Y$-a-t-il un $Q$ ou un $R$ ?). We anticipated that the exclusiveness evident in the Implicature-Potential Problem of Experiment 1 would be absent and that participants would consider the provided conclusion (Is there $a Q$ or an $R$ ?) to be compatible with an implied conjunctive conclusion (indicating an inclusive interpretation for or). Thus, when presented If $P$ then $Q$ and $R ; P$, participants ought to respond affirmatively to the question Is there a $Q$ or an $R$ ?

\section{Method}

Participants. A total of 20 Masters students from the Institut des Sciences Cognitives and the Université de Grenoble volunteered to participate. Only those who had no background in logic were included. Participants' backgrounds were mostly in fields related to the neurosciences and psychology.

Materials, design and procedure. This experiment was identical to Experiment 1, with the sole exception concerning the presented conclusions. These were now expressed, e.g., as Is there an R? or Is there a $Q$ or an R? etc. 
TABLE 6

Experiment 3

\begin{tabular}{|c|c|c|c|c|c|}
\hline \multicolumn{2}{|c|}{ Premises } & \multirow{2}{*}{$\begin{array}{l}\text { Conclusion } \\
\text { Is there a } \mathrm{Q} \text { or an } \mathrm{R} \text { ? }\end{array}$} & \multirow{2}{*}{$\frac{Y e s}{80 *}$} & \multirow{2}{*}{$\frac{N o}{20}$} & \multirow{2}{*}{$\frac{I I}{0}$} \\
\hline 1 & $\begin{array}{l}\text { If there is a } P \text { then } \\
\text { there is a } Q \text { and an } R \text {. } \\
\text { There is a P. }\end{array}$ & & & & \\
\hline 2 & $\begin{array}{l}\text { If there is a P or a Q } \\
\text { then there is an R. } \\
\text { There is a P. } \\
\text { There is a Q. }\end{array}$ & Is there an $\mathrm{R}$ ? & $90 *$ & 10 & 0 \\
\hline 3 & $\begin{array}{l}\text { If there is a } \mathrm{P} \text { or a } \mathrm{Q} \\
\text { then there is an } \mathrm{R} \text {. } \\
\text { There is a } \mathrm{P} \text { and a } \mathrm{Q} \text {. }\end{array}$ & Is there an $\mathrm{R}$ ? & $75 *$ & 15 & 10 \\
\hline
\end{tabular}

Percentage of participants responding "Yes", "No", or "Insufficient Information to decide (II)" to conclusions presented in question form in the Implicature-Potential problem (\#1), the Disjunctive Modus Ponens problem with implicitly conjunctive minor premises (\#2), and the Disjunctive Modus Ponens problem with an explicitly conjunctive minor premise (\#3) in Experiment 3. $N=20$.

$* p<.05$.

\section{Results and discussion}

As in Experiment 1, participants' responses to the standard problems (Problems 15-18) are similar to those in the literature and to those in Experiment 1. Moreover, there is no standard problem in Experiment 3 that is statistically different from its counterpart in Experiment 1. In fact, no problem is significantly different across experiments, with one exception: The Implicature-Potential problem in Question Form (\#1), which is shown below, yielded responses that indicate that the disjunction was treated inclusively.

If there is a $P$ then there is a $Q$ and an $R$.

There is a $\mathrm{P}$.

Is there an $\mathrm{Q}$ or an $\mathrm{R}$ ?

Eighty percent of participants responded "Yes" which is significantly above conservative predictions based on chance. Clearly, this percentage is significantly different from that found on the Implicature-Potential problem (with an asserted conclusion) in Experiment $1, \chi^{2}(1)=12.3, p<.001$ (see Table 6). This indicates that the question form serves as a linguistic context in which inclusive interpretations are acceptable. 


\section{EXPERIMENT 4}

Experiment 4 has two aims. First, it attempts to validate the findings from Experiments 1 and 3 in one overarching design, thus better justifying comparisons. That is, we want to verify that a participant's initial spontaneous interpretation to the original Implicature-Potential problem varies as a function of presentation form (Conclusion-as-assertion vs Conclusion-as-question). Second, we provide a more severe test to our hypothesis by going one step further: we present both sorts of materials to each participant, but in one of two different orders, so as to determine the extent to which the linguistic manipulation is offset by prior experience with the problem. Thus, all participants receive both sets of problems as they were presented in Experiments 1 and 3, but there are two groups, based on presentation order (Conclusion-asassertion first vs Conclusion-as-question first).

There are three possible patterns that could arise out of the investigation into order. The first, which would be strong support for our claims, is one in which a participant chooses to interpret $Q$ or $R$ inclusively when it is in a question form and to interpret it exclusively when it is asserted, regardless of order. This would indicate that participants are sensitive to the linguistic-pragmatic manipulation. A second would be consistent responses across the two encounters with the problem. Given the findings reported by Evans and Newstead across three blocks of trials on a standard disjunctive task, it appears that participants put a premium on consistency, so it would be relevant to know if this is the case even when pragmatic information intervenes.

Finally, it would be revealing if only the inclusive interpretation is open to change across the experiment. We have argued that the primary interpretation of or is inclusive and that it is open to an exclusive interpretation as a function of the implicature. If this is the case, one should see a one-way influence across the two sets of materials wherein the inclusive interpretation can be modified more readily than the exclusive interpretation. Those who receive the materials encouraging the inclusive interpretation first (the Conclusion-as-question) ought to be more likely to switch than those who first receive the materials encouraging the exclusive interpretation (the Conclusion-as-assertion). In a similar vein, an initial negative response to an assertion could influence the participant's response to a question because once the implicature is produced and adopted it would arguably be difficult to undo.

\section{Method}

Participants. A total of 35 students from the Université de Lyon volunteered to participate. Only those who had no background in logic were included. Participants' backgrounds were mostly in fields related to the social sciences and psychology. Three participants were dropped due either to computer or attention failure. 
Materials, design and procedure. This experiment was identical to Experiments 1 and 3, with three minor modifications. First, both sets were presented. Second, the order of the two sets was varied. Finally, the letters in one of the sets were modified to minimise the chance that participants would detect the similarity between the two sets.

\section{Results and discussion}

As in the previous experiments, none of the control problems yielded unusual responses and the remaining problems (other than the original ImplicaturePotential problem) prompted responses that were statistically comparable to their counterparts from the previous experiments (where problems in the Conclusionas-assertion condition were compared to Experiment 1 and the problems in the Conclusion-as-question condition were compared to Experiment 3 ).

Here, we focus only on the original Implicature-Potential problem. We conduct four chi-square analyses from the same data and thus reduce our level of significance to .0125 . As in the prior experiments, there were no Insufficient Information responses to this problem (in any of its versions) so even though an affirmative response serves as the dependent measure, negative responses could serve as well.

First, we compare spontaneous performance by analysing participants' first encounter with the problem before investigating participants' responses to both trials of the problem. Rates of spontaneous affirmative responses (i.e., at first encounters) were significantly higher among the Conclusion-as-question versions of the problem (.69) than among the Conclusion-as-assertion problems (.13), $\chi^{2}(1)=10.49, p<.01$. This is comparable to the findings gathered across Experiments 1 and 3. Unlike for Conclusions-as-assertions, participants' initial interpretation of disjunctive questions in the Implicature-Potential problem appears to be inclusive (see Tables 7 and 8 ).

In order to determine the role of prior experience on judgements, we categorise participants' responses into two Presentation Orders (Question-First vs Assertion-First) and four response patterns (1. Yes-to-Question/Yes-toAssertion; 2. Yes-to-Question/No-to-Assertion; 3. No-to-Question/Yes-toAssertion; 4. No-to-Question/No-to-Assertion). Results indicated that the observed distribution was different from one expected based on marginal totals, $\chi^{2}(3)=11.32, p<.0125$. As a description of individual differences will point out later, a strong majority of participants $(84 \%)$ applied the same response in their two encounters with the problem. This resulted in a significant difference in rates of affirmative responses to the Conclusion-as-question version when it was presented first as opposed to second, $\chi^{2}(1)=8.13, p<.0125$. There is a moderate, though non-significant, difference in rates of affirmative responses to the Conclusion-as-assertion version when it was presented first as opposed to second, $\chi^{2}(1)=3.86, p>$. 0125 . 
TABLE 7

Experiment 4: Type of conclusion $\times$ presentation order

\begin{tabular}{lcc}
\hline $\begin{array}{c}\text { Order in which } \\
\text { conclusion appeared }\end{array}$ & \multicolumn{2}{c}{$\begin{array}{c}\text { Conclusion type } \\
\text { Is there a } Q \text { or an R? }\end{array}$} \\
\hline There is a or an $R$ \\
When presented second & 69 & $13^{*}$ \\
\hline
\end{tabular}

Percentage of participants responding "Yes" to the implicature-Potential problem (If $P$ then $Q$ and $R ; P / / Q$ or $R$ ) as a function of the two different types of conclusion (Question vs Assertion) and as a function of presentation order in Experiment 4. 16 participants were presented 18 problems with Conclusions-as-questions before the same 18 problems (having different letters) were presented with Conclusions-asassertions. For 16 other participants, the order was reversed. Among both sets was the Implicature-Potential problem. $N=32$.

${ }^{*} p<.05$.

TABLE 8

Experiment 4: Response patterns

Yes-to-Question Yes-to-Question No-to-Question No-to-Question

Yes-to-Assertion No-to-Assertion Yes-to-Assertion No-to-Assertion

Total

Question

first/

Assertion

7

4

0

5

16

second

Assertion

first/

Question

2

1

0

13

16

second

Four different response patterns to the two Implicature Potential problems (If $P$ then $Q$ and $R ; P / / Q$ or $R$ ) in Experiment 4 based on two conclusion forms (Question and Assertion) and two presentation orders. $N=32$.

It appears that the linguistic-pragmatic manipulation led to its anticipated outcome as long as it was the participant's initial spontaneous interpretation. Once the experimental manipulation prompted a participant to adopt one of the two interpretations, he or she generally stayed with it, thus blocking the linguistic-pragmatic information from influencing responses on the second round. This demonstrates why it is important to our methodology to generally avoid repeated trials for problems that have two interpretations.

There is no strong statistical evidence indicating that responses are inclusive when the Conclusion-as-question problem is first and gravitate towards exclusiveness otherwise. Nevertheless, a brief overview of individualdifferences 
is warranted and it does show some gravitation towards exclusive interpretations. We point to three observations. First, the only inconsistent responses were among those who responded affirmatively to the Conclusion-as-question problem and negatively to the Conclusion-as-assertion problem (which means that only three patterns of response emerged - both affirmative, both negative, or affirmative to question and negative to assertion). Second, as the data indicate, a huge majority of participants give the same response (either both affirmative or both negative) across the two trials; whereas an affirmative response (indicating an inclusive interpretation) during a participant's first encounter with a problem leads to an impressive number of affirmatives in the second (9 participants, 28\%) an even higher percentage of participants stayed with negative responses (indicating an exclusive interpretation) across the two encounters (18 participants, $56 \%$ ). To make our third observation, we point to two contrasting findings: (a) whereas only 2 participants who received the Conclusion-asassertion problem first provided affirmative responses at both encounters (13\%), 5 participants who received the Conclusion-as-question version first provided negative responses to both encounters (31\%); (b) whereas there was a moderate chance that someone who initially responded affirmatively in the Conclusion-asquestion problem would switch to a negative response in the Conclusion-asassertion problem (4 out of $11,36 \%$ ), chances were low that someone who answered negatively to the Conclusion-as-assertion problem would switch to an affirmative response on the Conclusion-as-question problem (only 1 person out $14,7 \%$ ). Thus, out of the small number (5) who switched, 4 received the Conclusion-as-question problem first. All told, analyses of individualdifferences indicate that consistency was primary, exclusive interpretations were the more prominent of these, and, if there was a switch, it was likely in the direction of the exclusive.

\section{GENERAL DISCUSSION}

Most work in reasoning rightly takes for granted that disjunctions have two interpretations. Researchers usually dispense with this potential problem by anticipating or's two interpretations (e.g., see Johnson-Laird, Byrne, \& Schaeken, 1992) or by making clear which one is intended (e.g., Wason \& Brooks's [1979] THOG problem). However, few studies have tried to capture how $o r$ is understood when left on its own in minimally adorned contexts and, to our knowledge, there has been no empirical work that has aimed to predict with some confidence when to expect exclusive and inclusive interpretations of or as described here. The present work engaged the field of linguistic pragmatics in order to uncover situations in which such predictions are tenable.

We have argued that the more basic interpretation of or is inclusive and that listeners can detect rather easily, based on the speaker's (or in this case the experimenter's) intention, whether or not to employ the exclusive-or. The 
exclusive interpretation arises when a conclusion $Q$ or $R$ is more informative or relevant and is prompted by the implicature (but not both) which is added to the disjunction. The ease with which this could be done would explain why or in ordinary usage is so readily understood as an exclusive disjunction. We focused on one such situation (the Implicature-Potential Problem of Experiment 1) by employing a disjunction when a conjunction is implied (If $P$ then $Q$ and $R ; P / / Q$ or $R$ ). This problem showed that a significant percentage of participants treat the conclusion as exclusive. We investigated other disjunctive situations as well, such as when the disjunction is in the consequent of a conditional (If $P$ then $Q$ or $R$; $P / /$ not both $Q$ and $R$ ). The results from this problem also supported our hypothesis.

We also investigated two linguistic contexts that, according to recent linguistic literature, ought to lead to inclusive interpretations: (a) the antecedent of conditionals, and (b) question forms. The results were quite clear on three counts. First, one finds that disjunctions in the antecedents of conditionals generally lend themselves to inclusive interpretations. Second, the implicature that was salient in the original Implicature-Potential problem in Experiment 1 was significantly less evident once the conclusion was presented as a question in Experiment 3. Third, Experiment 4 replicated the finding concerning question forms as long as one considers participants' initial encounter with the problem.

The tasks in these experiments used arbitrary materials, which leads us to argue that there is no particular facilitation for one interpretation or the other that is due to the content of the syllogisms or to the storyline in which the premises were presented. The rates of exclusive interpretations are particularly impressive here, given that they are higher than in any of the previous studies that (a) presented problems in which both disjuncts were true and (b) used arbitrary materials (i.e., Braine \& Rumain, 1981; Evans \& Newstead, 1980; Paris, 1973). In this paper, we have argued that our relatively high rates of exclusiveness are linked to the relative ease of our tasks which allow more readily for the production of implicatures. A closer look at the other experiments supports this claim. In each of the three other studies, one finds at least one kind of complexifying element that could render its task more challenging than ours. Paris's study involves disjunctive (and other sorts of) statements that concern unrelated complex images, Evans and Newstead's includes a longer series of problems (48) involving effort-consuming negations, and Braine and Rumain indicate that the disjunctive statements used in their "box" problems made reference to two animals out of four and, thus, had distractors. Of course, a systematic investigation of relative difficulty and its link to implicatures would provide for stronger evidence for our claim and would make for a useful line of research in the future.

Whereas the role of pragmatics is appreciated as critical in the reasoning literature, especially for describing non-normative responses (e.g., Macchi, 1995; Politzer, 1986, Politzer \& Noveck, 1991; van der Henst, 1999), few take advantage of pragmatics in order to elucidate reasoning more generally as we do 
here (for a notable exception, see Sperber, Cara, \& Girotto, 1995). The present contribution is twofold. One is that the exclusive interpretation of $o r$, by way of the implicature but not both, arises when it is more informative (than an inclusive interpretation). The notable example being when or underdetermines an implied conjunctive conclusion. The other is that one can identify "negative" linguistic contexts (e.g., antecedents of conditionals and question forms) in which inclusive interpretations are considered appropriate because they render the utterance more informative. Remarkably, "negative" contexts that license inclusive interpretations appear to converge with those that license Negative Polarity Items like any.

Manuscript received 10 January 2001

Revised manuscript received 31 January 2002

\section{REFERENCES}

Bell, V., \& Johnson-Laird, P. (1998). A model theory of modal reasoning. Cognitive Science, $22(1), 25-51$.

Braine, M., \& Rumain, B. (1981). Children's comprehension of “or": Evidence for a sequence of competencies. Journal of Experimental Child Psychology, 31, 46-70.

Cherchia, G. (2001). Scalar implicatures, polarity phenomena, and the syntax/pragmatics interface. In A. Belleti (Ed.), Structures and beyond. Oxford: Oxford University Press.

Cohen, J.D., MacWhinney, B., Flatt, M., \& Provost, J. (1993). PsyScope: A new graphic interactive environment for designing psychology experiments. Behavioral Research Methods, Instruments, and Computers, 25(2), 257-271.

Evans, J.St.B.T., \& Newstead, S.E. (1980). A study of disjunctive reasoning. Psychological Research, 41, 373-388.

Fauconnier, G. (1975). Pragmatic scales and logical structure. Linguistic Inquiry, 6, 353-375.

Fillenbaum, S. (1974). Or: Some uses. Journal of Experimental Psychology, 103(5), 913-921.

Gazdar, G.A. (1979). Pragmatics: Implicatures, presupposition and logical form. New York: Academic Press.

Geis, M.L., \& Zwicky, A.M. (1971). On invited inferences. Linguistic Inquiry, 2, 561-566.

Horn, L. (1973). Greek Grice: A brief survey of proto-conversational rules in the history of logic. Chicago Linguistic Society, 9, 205-214.

Horn, L. (1989). A natural history of negation. Chicago: University of Chicago Press.

Johnson-Laird, P.N., Byme, R.M.J., \& Schaeken, W. (1992). Propositional reasoning by model. Psychological Review, 99, 418-439.

Johnson-Laird, P., Legrenzi, P., Girotto, V. \& Legrenzi, M.S. (2000). Illusions in reasoning about consistency. Science, 288, 531-2.

Ladusaw, W. (1979). Polarity sensitivity as inherent scope. Ph.D. Dissertation, University of Texas at Austin.

Levinson, S. (1983). Pragmatics. Cambridge: Cambridge University Press.

Macchi, L. (1995). Pragmatic aspects of the base rate fallacy. Quarterly Journal of Experimental Psychology, 48A(1), 188-207.

Newstead, S.E., Griggs, R., \& Chrostowski, J.J. (1984). Reasoning with realistic disjunctives. Quarterly Journal of Experimental Psychology, 36A, 611-627.

Noveck, I.A. (2001). When children are more logical than adults: Investigations of scalar implicature. Cognition, 78(2), 165-188. 
Noveck, I.A., \& Politzer, G. (1998). Leveling the playing field: Investigating competing claims concerning relative inference difficulty. In M.D.S. Braine \& D.P. O’Brien (Eds.), Mental logic. Hillsdale, NJ: Lawrence Erlbaum Associates Inc.

Paris, S. (1973). Comprehension of language connectives and propositional logical relationships. Journal of Experimental Child Psychology, 16, 278-291.

Pelletier, F.J. (1977). Or. Theoretical Linguistics, 4(1/2), 61-74.

Politzer, G. (1986). Laws of language use and formal logic. Journal of Psycholinguistic Research, $15,47-92$.

Politzer, G., \& Noveck, I.A. (1991). Are conjunction rule violations the result of conversational rule violations? Journal of Psycholinguistic Research, 20(2), 83-103.

Roberge, J.J. (1974). Effects of negation on adults' comprehension of fallacious conditional and disjunctive reasoning abilities. Journal of General Psychology, 91, 287-293.

Roberge, J.J. (1976a). Effects of negation on adults' disjunctive reasoning abilities. Journal of General Psychology, 94, 23-28.

Roberge, J.J. (1976b). Reasoning with exclusive disjunction arguments. Quarterly Journal of Experimental Psychology, 28, 419-427.

Sperber, D., Cara, F., and Girotto, V. (1995). Relevance theory explains the selection task. Cognition, 57(1) 31-95.

Sperber, D., \& Wilson, D. (1985/1996). Relevance: Communication and cognition. Oxford: Basil Blackwell.

van der Henst, J.B. (1999). The mental model theory of spatial reasoning re-examined: The role of relevance in premise order. British Journal of Psychology, 90(1), 73-84.

van Duyne, P.C. (1974). Realism and linguistic complexity in reasoning. British Journal of Psychology, 65, 59-67.

Wason, P.C., \& Brooks, P.G. (1979). THOG: The anatomy of a problem. Psychological Research, $41,79-90$. 